\title{
Wie erreichen die Kirchen den einzelnen? \\ Überlegungen zu einer missionarischen Aufgabe
}

\author{
von Henk Hoekstra
}

\section{Kirchliche Kommunikationsprobleme}

\subsection{Die Botschaft der Kirche und die Realität der Kommunikation}

Überlegungen zum evangelisch-missionarischen Aspekt der Frage, wie die Kirchen den einzelnen erreichen, beschäftigen sich mit einem sehr vielschichtigen Problem. Die Kirchen sehen es als ihren selbstverständlichen Auftrag an, dem Menschen von heute die Botschaft Christi zu verkünden. Sie sehen sich als Verkäufer einer religiösen Heilsbotschaft. In der Kommunikation dieser Heilsbotschaft aber ergeben sich ernsthafte Schwierigkeiten. Realität und Erfahrungen zeigen, daß diese Kommunikation der Kirchen heute mit wenigen oft nur schwer und mit vielen fast gar nicht gelingt. Deswegen ist es verständlich, daß man immer wieder danach fragen muß, wie die Kirchen den einzelnen erreichen können.

\subsection{Zugangsmöglichkeiten zur Analyse kirchlicher Kommunikationsprobleme}

Für die Frage der Kommunikation der Kirchen gibr es sehr unterschiedliche Zugänge. Man kann sich z. B. fragen: Liegen die Kommunikationsschwierigkeiten vielleicht beim einzelnen, den man nicht erreichen kann? Warum läßt er sich nicht erreichen? Was könnte oder sollte getan werden, um ihn „erreichbar" zu machen? (Empfängeraspekt). Liegen die Kommunikationsprobleme möglicherweise mehr in der Botschaft selbst, weil sie inhaltlich als nichtssagend oder bedeutungslos erfahren und kommunikativ so gestaltet wird, daß sie für den einzelnen von heute als unbedeutend und unattraktiv erscheint? (Botschaftsaspekt). Oder liegen die Kommunikationsprobleme vielleicht mehr beim Sender, weil dieser unglaubwürdig oder der Lebenswelt des Empfängers fremd ist oder sich generell unkommunikativ verhält? (Senderaspekt). Oder liegen die Kommunikationsprobleme vielleicht in den Beziehungen zwischen Sender und Empfänger, weil der Sender unfähig ist, mit diesem Empfänger authentische Beziehungen herzustellen, weil er religiös nicht ansprechbar ist, so daß beide sich beziehungslos gegenüber stehen? (Beziehungsaspek $t$ ).

Oder liegen die Kommunikationsschwierigkeiten vorwiegend im heutigen gesellschaftlichen Kontext, in welchem die Institution Kirche für die meisten Menschen nicht mehr zählt, als überflüssig oder gar (auf Grund früherer Erfahrungen) als negativ und schädlich empfunden wird? (Kontextaspekt). So gesehen ist die Frage nach dem Kommunikationsverhältnis der Kirchen gegenüber dem einzelnen vielschichtig. Dabei muß man sich allerdings wohl darüber klar sein, daß sich diese Frage nicht auf einen einzelnen der genannten Aspekte allein zurückführen läßt. Sie gehören wahrscheinlich alle zusammen und beeinflussen sich gegenseitig.

Henk Hoekstra ist nach kommunikationswissenschaftlichen Studien an der Universität Löwen und einer Tätigkeit an der Katholischen Theologischen Hochschule Amsterdam in der Kommunikations- und Medienerziehung tätig und Mitarbeiter des "Katholieke Radio Omroep ${ }^{*}$ (KRO) Hilversum. Vorliegender Beitrag ist die Bearbeitung eines Referats auf der Jahrestagung 1982 des Okumenischen Arbeitskreises für Information in Europa, Bossey Genf (22.-25. März 1982). 
Der Zugang zu diesen Fragen in kommunikationswissenschaftlicher Sicht soll hier versucht werden, auch wenn dabei keine Vollständigkeit erreicht werden kann. Die kirchliche Kommunikation mit dem einzelnen wird in ihren Möglichkeiten und Grenzen sehr stark yom heutigen gesamtgesellschaftlichen Kontext und dessen Bedingungen mitbestimmt. Deswegen soll hier auch zunächst dieser gesellschaftliche Kontext und die Situation des einzelnen herausgearbeitet werden. Dann folgen unter dem Stichwort "Die Kirchen in Kommunikation mit dem einzelnen" ein Kommunikationsmodell, es werden die Grundlagen eines kirchlichen Kommunikationskonzeptes skizziert und die kommunikativen Dimensionen der kirchlichen Heilsbotschaft angesprochen.

\section{Gesellschaftlicher Kontext und Lebenssituation des einzelnen}

\subsection{Gesellschaftlicher Kontext}

2.1.1 Die geistige Situation heute

Wir befinden uns heute in gesellschaftlichen und kulturellen Änderungsprozessen, die zugleich eine geistige Krise aufzeigen. Diese Änderungsprozesse beeinträchtigen tiefgehend die gesellschaftliche und kirchliche Kommunikationssituation. Die Kirchen haben zuerst und vor allem mit der christlichen Weltsicht, Sinndeutung und Wertbestimmung des menschlichen Lebens und Zusammenlebens zu tun. Gesellschaftlich-kulturell zeigt sich gerade in dieser Weltsicht und der Wertbestimmung das Kernproblem der heutigen Krise.

Kennzeichnend für das Bild der heutigen Gesellschaft ist, daß kollektive Sinnerfahrung, Sinnfindung und Sinndeutung gesellschaftlich und individuell zu einer ernsthaften Schwierigkeit geworden sind. Die Menschen von heute haben mehr als frühere Generationen, die in einer anderen Gesellschaft und Kultur lebten, Mühe mit diesem Erfahren und Finden eines Lebenssinnes". Welche Entwicklungen haben sich in dieser "neuen“ Gesellschaft und Kultur unserer Tage gezeigt? Wie läßt sich die heutige Situation des einzelnen charakterisieren? De Valk erwähnt je fünf Entwicklungen im Bereich der Ideen und der sozialen Prozesse, die das gesellschaftliche Leben bestimmen $^{2}$ :

Im Bereich der Ideen verweist er auf folgende Tatsachen:

1. Einen Säkularisierungsprozeß, der Lebenserscheinungen primär als Naturerscheinungen betrachtet, in denen kein Sinn liegt, sondern die nur als Zufälle und Unvermeidlichkeiten gewertet werden.

2. Die instrumentelle Lebenshaltung, die den Sinn des Handelns nur im Nutzen durch Rationalisierung des Lebens in allen Lebensbereichen sieht.

3. Die Zunahme einer individualisierten und privatisierten Lebenshaltung, in deren Folge der Mensch sich selbst mehr als ein selbständiges und autonomes Individuum und nicht primär als Mitglied von vielerlei gesellschaftlichen Gruppierungen sieht, für die er mitverantwortlich ist.

4. Die Entfaltung individueller Veranlagungen und Möglichkeiten. Der Mensch zieht sich mehr und mehr in seine private Sphäre zurück. Was das Persönliche übersteigt, wird nicht geschätzt und gewertet.

5. Den kulturellen Pluralismus, gekennzeichnet durch konkurrierende Sinngebungssysteme. Folgen sind die Relativierung der Weltanschauungen (Relativismus) und die Abnahme der Einbindung in weltanschauliche Gruppierungen. 
Im Bereich der sozialen Prozesse sieht De Valk folgende Entwidklungen:

1. Der Sinn des Lebens wird größtenteils erfahren im „Hören-Sagen“. In fast allen Gesellschaften war der Sinn des Lebens Sache einer kleinen Gruppe „weltanschaulicher Experten", die stellvertretend und vermittelnd sich mit dieser Frage beschäftigten. Heute sind diese Experten sehr verteilt, und es bildet sich ein Heer von "Führern“. Dabei wird jeder einzelne mehr und mehr sein eigener weltanschaulicher Experte, mit vielen Problemen der Unsicherheit und Verwirrung.

2. Eine sehr differenzierte Arbeitsteilung in Großorganisationen fordert vom Menschen spezialisierte Teilarbeit und versperrt die Sicht aufs Ganze. Die Gesellschaft ist deswegen einerseits rationalisiert, andererseits aber zeigen sich in ihr viele irrationale Züge. Es gibt zielorientiertes Erfüllen von Aufgaben (funktionale Rationalität) aber die Beurteilung des Sinnes dieser Aufgaben fehlt (substantielle Rationalität).

3. Eine Schwächung der Institutionen als Folge von Individualisierung und Privatisierung und die Abnahme der Bedeutung führender Eliten ist festzustellen. Daraus folgt eine abnehmende Bedeutung von überpersönlichen Institutionen für die gesellschaftliche Sinngebung.

4. Rasche soziale Änderungen, vor allem das Tempo dieser Änderungen erschweren die Sinnerfahrung, bedrohen die notwendige Stabilität und den Halt, Unsicherheit und Relativismus berühren die Sinngebung für menschliches Leben negativ.

5. Bei der Uberflutung mit unstrukturierter Information und Unterhaltung durch die Massenmedien wird fast alles gleich wichtig und interessant dargestellt und allen Dingen unbesehen gleicher Sinn und Wert beigemessen.

Unter dem Einfluß dieser Entwicklungen hat sich die Situation der Kirche grundlegend geändert. In früheren Generationen hatten die Kirchen das Monopol der Sinndeutung und Sinngebung, der Weltauslegung und Wertbestimmung.

Jetzt sind sie eines der vielen gesellschaftlichen Teilsysteme geworden, die mit vielen anderen Deutungssystemen in Konkurrenz treten müssen. Trotzdem haben die Kirchen heute öffentlich noch eine Rolle für die Sicherung der Identität des einzelnen und der Legitimierung von Handlungsnormen. Die Kirchen stehen außerdem heute in der Pflicht, diese Legitimität zu belegen, ihre Zuständigkeit für Sinnfragen, für letzte Werte und Kontingenz zu begründen ${ }^{3}$, d.h. die Kirchen müssen die Bedeutung ihrer Heilsbotschaft öffentlich, im Dialog mit der Gesellschaft und den einzelnen aufzeigen.

\subsection{Die Lebenssituation des einzelnen}

Bis vor wenigen Jahrzehnten waren Leben und Zusammenleben für den einzelnen wohl einfacher und unproblematischer als heute: Gemeint sind Erziehung und Schule, Kirche und entsprechende Bezugsgruppen, wie politische Parteien, Vereine und Gewerkschaften, die ganz bestimmte Anschauungen, Wertvorstellungen und Maßstäbe für die Bewältigung des Lebens entwickelten, welche die Welt strukturierten, durchschaubar und greifbar machten. Ihre Normen sagten, was gut und richtig, was schlecht und falsch war. Der einzelne wußte für fast alle Lebensbereiche, wie zu denken, zu urteilen, und was zu tun und wie zu handeln war. Leben und Zusammenleben waren „vorgegeben“, und soziale Wegweiser wurden für das Leben „mitgegeben “. In dieser Welt gab es wenige Unsicherheiten und Zweifel, und konkrete Alternativen gab es selten. Abweichungen wurden bei dieser Mentalität kaum toleriert, denn die soziale Kontrolle mit positiven und negativen Sanktionen war stark ausgeprägt ${ }^{4}$. Dies heißt allerdings nicht, daß damals das Leben und Zusammenleben völlig unproblematisch 
und konfliktlos war. Selbstverständlich gab es unterschiedliche Weltanschauungen, Wertsysteme, unterschwellige oder ausgesprochene Spannungen, Interessenkollisionen und Konflikte. Innerhalb einer Gruppe aber wußte man genauer, wie diese Probleme $\mathrm{zu}$ bewerten und Standpunkte zu bestimmen waren, denn man hatte sich in eigenen Gruppierungen zusammengeschlossen und konnte sich so einander bestätigen. Der Auseinandersetzung nach außen konnte man so aus dem Wege gehen. Auf diese Weise konnten Erziehung und Schule, Kirchen und Bezugsgruppen gruppenspezifische Anschauungsformen und Wertvorstellungen ziemlich einheitlich weitergeben.

Dadurch war eine Lebensbewältigung einfacher und die Frage nach Sinngebung und Orientierung wurde kaum bewußt gestellt.

Heute kann sich der einzelne nicht mehr in gleichem Maße auf vorhandene kollektive Deutungsschemata größerer Bezugsgruppen stützen. Die Bewältigung des Lebens ist schwieriger und eine bewußte Lebensorientierung notwendiger geworden. Der einzelne hat heute wohl weniger Klarheit und Halt, mehr Zweifel und Unsicherheit, aber auch mehr Chancen. Allerdings erfordert das heutige Leben im Pluralismus vom einzelnen mehr bewußte und aktive Beweglichkeit ${ }^{5}$. Das heutige Leben erweitert den primären und vor allem sekundären Erfahrungshorizont und steigert das Problembewußtsein. Hier spielen die Medien eine große Rolle. Durch diese tiefgehenden Anderungen wird der einzelne gezwungen, seine Welt mehr aus eigenen Kräften zu erbauen, seine eigenen Orientierungspunkte zu wählen, seine Weltanschauung zu klären, seine Werte zu gewichten und diese in seinem Lebensstil zum Ausdruck zu bringen. Er hat mehr Möglichkeiten der Wahl, wird dadurch aber auch zugleich zur persönlichen Entscheidung und Standortbestimmung gezwungen ${ }^{0}$ : "Each individual faces the task of continually reorganising, remaking and relating his internal and his external worlds ${ }^{\alpha 7}$.

Diese Lebenssituation belastet den einzelnen kognitiv und emotional. Viele Menschen fühlen ihre Ich-Identität durch diese Anforderungen zu einer kognitiven und normativen Mobilität bedroht. Sie sind diesen Anforderungen nicht gewachsen und fühlen sich überfordert. Viele lassen sich auf den Wellen pluralistischer Lebenswelt hin und her treiben oder greifen zurück auf die Autorität des starken Mannes, weil sie - ohnmächtig und unfähig zur persönlichen Standortbestimmung und zur persönlichen Lebensgestaltung - sonst in ein existentielles Vakuum geraten würden, das Frankl ${ }^{8}$ wie folgt umschreibt: „Im Gegensatz zum Tier sagen dem Menschen keine Instinkte, was er muß, und im Gegensatz zum Menschen von gestern sagen dem Menschen keine Traditionen mehr, was er soll. Nun, weder wissend was er muß, noch wissend was er soll, scheint er nicht mehr recht zu wissen, was er will. So will er denn nur, was die anderen tun - Konformismus! Oder aber er tut nur das, was die anderen wollen von $i$ mm wollen - Totalitarismus."

Die einzige Alternative sieht Frankl darin", daß er sich zu einer Weltanschauung durchdringt und auf dieser Basis sein Leben sinnvoll gestaltet: „Sinn kann nicht gegeben, sondern muß gefunden werden ... Sinn muß gefunden, kann aber nicht erzeugt werden ... Sinn muß aber nicht nur, sondern kann auch gefunden werden." Andere - einzelne, Gruppen, Institutionen - können dazu beitragen, vor allem auch die Kirchen.

\section{Die Kirchen in Kommunikation mit dem einzelnen}

Die Darstellung des gesellschaftlichen Kontextes und der Lebenssituation des einzelnen zeigt, wie seine existentiellen Fragen eine mögliche Antwort in der Heilsbotschaft der 
Kirchen finden können. Vor dem Hintergrund des Pluralismus beinhaltet diese Botschaft eine der vielen Möglichkeiten zur (gläubigen) Weltdeutung und Lebensanschauung, zur Existenzbewältigung und Zukunftserwartung, zur Sinngebung und Sinnerfahrung. Gerade wegen der Konkurrenz der Sinngebungssysteme untereinander müssen die Kirchen heute ihre Heilsbotschaft öffentlich artikulieren, um so die Aufmerksamkeit des einzelnen zu erreichen. Dies ist Teil der (neuen) Offentlichkeitsaufgabe der Kirchen. Sie haben es hier auch beim einzelnen nicht einfach. Gründe für die Schwierigkeiten sind u. a. die belastete Vergangenheit der Kirchen, ihr negatives Image und die Indifferenz des heutigen Menschen ihnen gegenüber. Sie sind deswegen in ihrer Kommunikation mit den einzelnen an Voraussetzungen gebunden, die sie beachten müssen. Vom kommunikationswissenschaftlichen Standpunkt aus sollen deswegen hier - zurückgreifend auf die anfangs erwähnten Aspekte der Kommunikation - einige Orientierungshinweise und Anhaltspunkte gegeben werden, die helfen, den einzelnen zu erreichen.

\subsection{Der kommunikative Einflußprozeß auf den einzelnen}

Braehmer ${ }^{10}$ hat die Literatur der kommunikativen Beeinflussung von Individuen untersucht und in einem Modell dargestellt. Dieses Modell skizziert die Bedingungen und Voraussetzungen, denen ein Sender in der Kommunikation mit einzelnen Rechnung zu tragen hat. Es beinhaltet fünf Faktoren:

1. Die Kommunikationsbotschaft stößt auf Prädispositionen des Empfängers, ruft dementsprechende interne Prozesse bei ihm hervor und bewirkt so bestimmte Effekte;

2. dieser Kommunikationsprozeß findet in gesellschaftlichen Kontexten statt und wird dadurch mitgeprägt; 3 . die Wirkung einer Kommunikationsbotschaft ist damit nicht nur abhängig vom Sender und seiner Botschaft, sondern auch von den Prädispositionen des Empfängers; 4. dieser Empfänger ist kein unbeschriebenes Blatt, er ist aktiv und bestimmt die Wirkungen mit; 5. seine Lebensgeschichte, Vorerfahrungen, Bedürfnisse, Erwartungen, Ideale und Werte, Ziele und aktuelle Situation, seine Gruppenzugehörigkeit und Beziehungen mit anderen Menschen intervenieren zwischen der Botschaft und der Wirkung. Auch die Kirchen haben in ihrer Kommunikation mit den einzelnen diese Faktoren zu berücksichtigen, wenn sie wirkungsvoll kommunizieren wollen.

Diese Darstellungen des gesellschaftlichen Kontextes, der Lebenssituation des einzelnen und des kommunikativen Modells, beinhalten Hinweise, Orientierungspunkte und Anhaltspunkte für ein mögliches Kommunikationskonzept der Kirchen, um den einzelnen zu erreichen. Gottschlich ${ }^{11}$ erwähnt - übrigens im Rahmen eines Aufsatzes über kirchlich-religiöse Werbung - die folgenden Komponenten eines solchen kirchlichen Kommunikationskonzeptes.

Psychisch-geistige Situation

Wenn die Kirchen von der geistig-psychischen Situation des einzelnen ausgehen und kommunikative Impulse zu fundamentalen Fragen menschlicher Existenz zum Inhalt machen, gelingt es eher, die Indifferenz gegenüber den Kirchen zu überwinden und Sinnfindungsprozesse auszulösen. Dabei müssen die Kirchen vor allem an die individuell und subjektiv unterschiedlichen Voraussetzungen des einzelnen anknüpfen. Paulus kann auch hier als Wegweiser dienen: In 1 Kor 9, 19-23, zeigt er sich nicht als ,allen alles" mit einer allgemeinen Botschaft, aus der jeder Empfänger für sich das Seine holen kann. 
Er ist Jude mit den Juden, Heide mit den Heiden, Schwacher mit den Schwachen, um die Menschen zu gewinnen und $\mathrm{zu}$ retten. Die Kirchen sollten deswegen im jeweils Spezifischen des einzelnen die Chancen der kommunikativen Erschließbarkeit des christlichen Glaubens suchen. Daher müssen sie sich mehrerer Kommunikationsstrategien bedienen und eine Typologie von Zielpersonen herausarbeiten, damit der einzelne in seiner besonderen Lage angesprochen werden kann. Nur so kann die Kirche ihre Möglichkeiten gegenüber den einzelnen wahrnehmen und einen Zugang finden, mit dem das kirchliche Heilsangebot als Sinnfindung erlebbar wird. Die Bedingungen zu kommunikativer Sinnverwirklichung befinden sich im einzelnen Empfänger und müssen daher auch von ihm her erschlossen werden. Sie liegen in seiner psychisch-geistigen Situation und in der subjektiven Funktion der kirchlichen Botschaft für den einzelnen. Dies erfordert die Fähigkeit, christliche Glaubenswirklichkeit entsprechend den individuellen Bedürfnissen des Empfängers zu thematisieren. Die Kirche soll nicht zuerst fordern, sondern geben. Der Empfänger muß deshalb als einzelner im Mittelpunkt stehen, und die Kirchen müssen bei seinen Bedürfnissen ihren Ausgangspunkt suchen.

\section{Typologie der Zielpersonen}

Die Kirchen sollten Kriterien entwickeln, die helfen, durch Thematisierung Publikum herzustellen: es bedarf einer Typologie, die aus den unterschiedlichen Arten des $\mathrm{Zu}$ gangs zum christlichen Glauben erwächst. Es ist eine Typologie, die aus den Gemeinsamkeiten der psychisch-motivationalen, bedürfnisspezifischen Ausgangssituationen kirchlich-religiösen Themen gegenüber basiert. Inhaltlich schließt dies jeden einzelnen ein. Gottschlich ${ }^{12}$ gründet einen Versuch zur Typologie auf den individuellen Voraussetzungen und Bedingungen zu kirchlich religiösen Themen. Folgende Skizze dieser Typologie konkretisiert und verfeinert, was hier gemeint ist:

Typus A:

Glaube als Abenteuer: dieser Typus umfaßt diejenigen Menschen, die durch Beispiele bedingungslosen Engagements angesprochen werden können (z. B. Heilige). Diese Gruppe - vor allem auch Jugendliche - ist aufgrund ihrer Einsatzfreude bei gleichzeitig starkem Sinnbedürfnis dem Fanatismus von Sekten aufgeschlossen. Sie wäre daher besonders unter Berücksichtigung der emotionalen Komponenten christlichen Glaubens anzusprechen. Dieser Zugang über den Weg der Hingabe bedarf einer Kommunikationsstrategie, die sich auszeichnet durch Ausklammerung von (allzuviel) Reflexivität und Relativierung. Im Vordergrund muß die Faszination des Bekennens stehen: Einschichtigkeit der Daseinsbetrachtung und Dominanz der Emotionen.

Typus B:

Das Numinöse am Glauben: dieser Typus umfaßt jene Gruppen, deren Zugang über das Geheimnisvolle und dessen Auswirkung auf einzelne und Menschheit gefunden werden kann. Die Darstellung von Wundern, von nicht erklärbaren Daseinsphänomenen wird hier dem Bedürfnis nach Außergewöhnlichem Rechnung tragen und kommunikative Anknüpfungspunkte bieten.

\section{Typus C:}

Glaube als Ausdruck von Vernünftigkeit: eine immer größer werdende Gruppe von Menschen sucht einen rationalen Zugang zum Glauben und zu Glaubenswerten. Im Unterschied zu Typus A steht bei diesem Typus das Warum im Vordergrund. Thematische Anknüpfungspunkte könnten hier die Darstellung und Begründung der Zweckmäßigkeit der Schöpfung im Rahmen eines zusammenhängenden Bauplanes sein (Bei- 
spiele von Naturwissenschaftlern). Diese Aufklärung über das Warum soll sich auch auf die Kirchen selbst beziehen. Die Zweckmäßigkeit und der Sinn bestimmter Entscheidungen und Handlungen kirchlicher Entscheidungsträger und die Grundlagen ihrer Entscheidungen müssen aufgewiesen werden, wenn man diese Zielgruppe ansprechen will.

\section{Typus D:}

Asthetische Dimension des Glaubens: diese Gruppe ist durch die ästhetischen Dimensionen des Glaubens und seiner Zeugnisse anzusprechen. Durch die Mitteilung der kulturellen, künstlerischen Erscheinungsbilder des Glaubens und der Religion ließe sich der Zugang zur jenseitigen, diese irdische Welt transzendierenden Welt eröffnen. Gegenstand und Form der kommunikativen Vermittlung müssen hier der dominant ausgeprägten und differenzierten Erlebnisfähigkeit, dem ästhetischen Anspruch Rechnung tragen.

\section{Typus E:}

Soziales Engagement als Leitmotiv des Glaubens: für diese Gruppe bedeutet Leben für Gott, für sich aus dem Glauben zuerst und vor allem soziales Engagement zu finden in den unterschiedlichen Lebensbereichen wie Entwicklungshilfe, Dritte Welt, Kranke, unterdrückte und ausgebeutete Menschen und Gruppen. Als Ausgangspunkt und kommunikative Zugänge bieten sich hier die Thematisierung der sozialen Leistungen der Kirche, einzelner Christen und Gruppen. Darüber hinaus muß die Perspektive auch auf die mit der Lehre Christi verbundene Spiritualität gerichtet werden, um vom Verständnis für den Nächsten zum Verständnis der transzendenten Bezüge zu kommen.

In diesen Grundlagen für ein mögliches Konzept der Kirchen zur Kommunikation mit den einzelnen wurde bisher vor allem der gesellschaftliche Kontext, der Empfängerund Botschaftsaspekt herausgearbeitet. Wenn die Kirchen in ihrer Kommunikation mit den einzelnen diese Punkte berücksichtigen, hat dies wichtige Konsequenzen für sie selbst als „Sender“. Glaubwürdigkeit, Attraktivität und gutes Image des Senders Kirche steigern Erleben und Reagieren des Empfängers. Die Kirchen werden an Bedeutung für die Menschen von heute gewinnen. Auf der anderen Seite aber fordert diese Art der Kommunikation von den Kirchen gegenüber den Empfängern und den Botschaften auch, sich selbst zu ändern. Es verlangt ein grundlegendes Uberdenken und mögliche Anderung des Selbstverständnisses der Kirchen.

Hier sind dann auch die kommunikativen Dimensionen der kirchlichen Botschaft zu berücksichtigen.

\subsection{Kommunikative Dimensionen kirchlicher Botschaft}

Schulz von Thun ${ }^{13}$ zeigt auf, daß, jede Kommunikationsbotschaft von der Seite des Senders betrachtet vier verschiedene Dimensionen hat. Diese Aspekte der Kommunikationsbotschaft rufen entsprechende Reaktionen beim Empfänger hervor. Für das Verstehen und Gelingen der Kommunikation der Kirchen mit den einzelnen ist deswegen sein Schema von großer Bedeutung:

Der Selbstoffenbarungsaspekt sagt aus, daß nicht Angst oder Verbergen, nicht Fassade oder Imponieren, sondern Kongruenz, Offenheit und Authentizität für das Gelingen kirchlicher Kommunikation mit dem einzelnen im Mittelpunkt stehen sollte.

Der Sachaspekt meint eine sachliche und verständliche Information, die eine volle Aufmerksamkeit für die Kommunikation verlangen. Diese Information soll auf die Erwartungen und Bedürfnisse der einzelnen Zielpersonen zugeschnitten sein. 
Inhalt/Thema

(worüber Sender informiert)

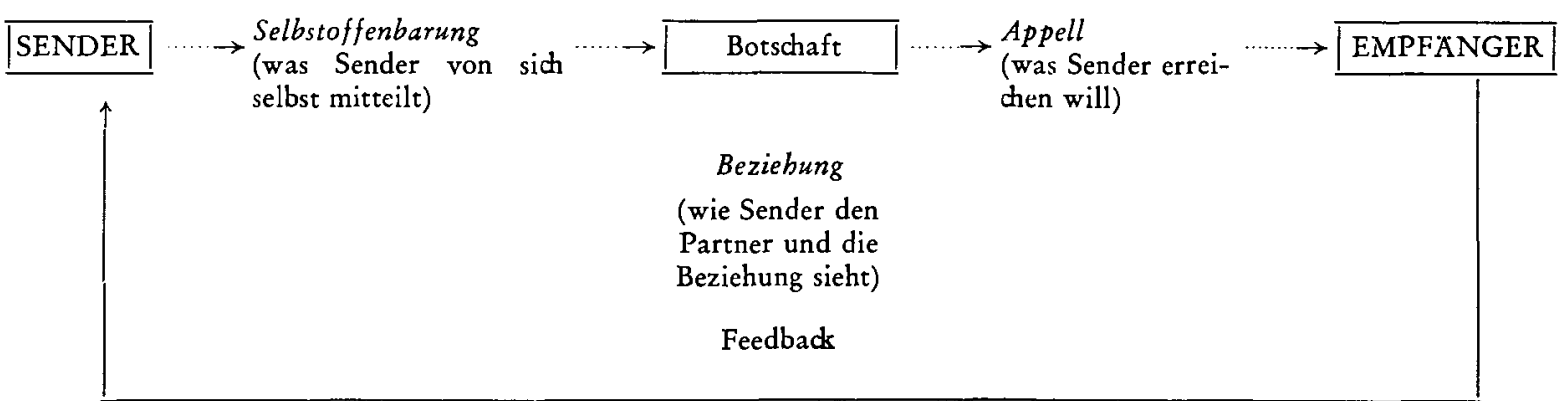

Selbstoffenbarung

Sach/Inhalt-

Beziehungs-

Appell

\section{Selbstoffenbarung}

1. Selbstoffenbarungsangst (Beurteilung, Richter)

2. Selbstdarstellung und Selbstverbergung (Imponier- und Fassadetechniken

3. Selbstpräsentation

(Kongruenz, Offenheit, Authentizität)

\section{Inhalt/Thema}

1. Sachlichkeit (Aufs Thema bezogene Information.

Vorrang von Störungen)

2. Verständlichkeit (Einfachheit, Struktur, Prägnanz, Stimulanz)

\section{Beziebung}

1. Verhaltenskreuz (Bevormundung, Geringschätzung, Hochachtung, Selbstbestimmung)

2. Fremdbild

3. Selbstbild

4. Beziehungsdefinition (Akzeptanz, Passivität, Ablehnung, Ignoranz)

\section{Appell}

1. Ausdrudk - Wirkung

2. Verborgene Appelle

3. Paradoxe Appelle

4. Offene, informative Appelle 
Der Beziehungsaspekt bedeutet, daß die Pflege von Beziehungen für die Kommunikation der Kirchen von großer Wichtigkeit ist. Denn die Pflege dieser Beziehungen, d. h. wie die Kirchen den einzelnen sehen und mit ihm umgehen, ist Voraussetzung und Bedingung für das Gelingen guter Kommunikation.

Der Anrufaspekt bedeutet, daß die Benutzung von Appellen nicht zunächst emotional, sondern informativ und offen sein soll. Die eigene Verantwortung des Empfängers muß berücksichtigt und geachtet werden. Deswegen dürfen diese Appelle nicht verborgen oder manipuliert sein.

Jeder Empfänger hört mit vier Ohren zu. Er ist besonders empfindlich für die Selbstoffenbarung, die Beziehungspflege und die Appelle kirchlicher Kommunikatoren.

Wenn die Kirchen in ihrer Kommunikation mit dem einzelnen vor allem diesen drei Aspekten größere Sorgfalt widmen, werden sie den einzelnen als Person erreichen, weil er sich dann eher öffnet für die christliche Botschaft der Kirche.

\section{Literatur}

Bennis, W. G., Benne, K. D., Chin, R.: The planning of change. New-York ${ }^{2} 1966$.

Berger, P. L., Berger, B., Kellner, H.: The homeless mind. New-York, 1973.

Bradford, L. P.: The teaching-learning transaction. In: W. G. Bennis, K. D. Benne, R. Chin: The planning of change. New-York, ${ }^{2} 1966$, S. 495.

Braehmer, U.: Leitsätze für die Kommunikationspraxis. Kommunikationswirkungen und publizistische Beeinflussung. Ein Modell zur Wirkungsforschung. In: „Publizistik", 25: 1981 1, S. 24-50.

J. M. M. De Valk: Maatschappelijke aspecten van de zinervaring: In: Zinervaring in de hedendaagse cultuur. "Annalen van het Thijmgenootschap" 70:1982 1. Amboboeken Baarn, S. $17 \mathrm{ff}$.

Frankl, V. E.: Der Mensch vor der Frage nach dem Sinn. München-Zürich 1979.

Gottschlich, M.: Kirche und Offentlichkeit: Zu den Grundlagen eines Konzepts kirchlich-religiöser Werbung. In: „Communicatio Socialis“, 13: 1980 3, S. 241-249.

Hall, B. P.: The development of consciousness. A confluent theory of values. New-York 1976.

Kappenberg, B.: Kommunikationstheorie und Kirche. Grundlagen einer kommunikationstheoretischen Ekklesiologie. Frankfurt-Bern, 1981.

Meiden, van der, A.: Mensen winnen. De overdracht van de boodschap. Baarn 1973.

O'Neill, N. \& G.: Shifting gears. Finding security in a changing world. New-York 1974.

Schulz von Thun.: Miteinander reden: Störungen und Klärungen. Psychologie der zwischenmenschlichen Kommunikation. Rororo-Sachbuch 7489. Reinbek bei Hamburg 1981.

Thung, M. A.: Missionaire Kerk, waarom en hoe? In: Rondom het Woord. Geloof, Wetenschap, Toeksomst. Theologische Etherleergang. 21: 1979 3, S. 51-59.

\section{Anmerkungen}

1 Berger: a. a. O. 1973.

2 De Valk: a. a. O. 1981.

${ }^{3}$ Kappenberg, 1981, $137 \mathrm{ff}$.

4 Bennis: a. a. O. 1966.

5 Berger: a. a. O. 1973.

6 Vgl. Hall, 1976; O’Neill, 1975.

7 Bradford: a. a. O. 1966, 495.

8 Frankl: a. a. O. $1979,142$. 
- Frankl: ib. 146.

10 Braehmer, 1981, $24 \mathrm{ff}$.

11 Gottschlich, 1981, $241 \mathrm{ff}$.

12 Gottschlich, 1981, $244 \mathrm{ff}$.

13 Schulz von Thun, 1981, 24 ff., 44 ff.

\section{SUMMARY}

Communications sciences can help the Churches to show how to reach man. The context of society and the living situation of any person conditions and influences the communication of the Churches with the People. For the Christian message to have access and to be delivered to people, the Churches should recognise the specific needs and desires of these people. From this aspect one can develop and refine a typology of the public.

Finally the Churches should not only try to be understandable with their messages but should also be open as a sender to the desires of the receiver and be aware of the appeal of their messages. These dimensions, to a great extent, determine the impact of the Christian message.

\section{RESUME}

Les sciences de la communication peuvent montrer aux Eglises comment atteindre l'homme. Le contexte social et la situation de vie du particulier conditionnent et influencent la communication des Eglises avec le peuple. Pour que le message chrétien ait accès et soit délivré au peuple, les Eglises doivent reconnaître ses besoins et désirs. Sous cet aspect, on peut développer et définir une typologie du public.

Finalement, les Eglises devraient non seulement essayer d'être compréhensibles avec leurs messages, mais aussi être ouvertes comme émetteurs aux désirs des recevants et être conscientes de l'intérêt de leurs messages. Ces dimensions déterminent en grande partie l'impact du message drétien.

\section{RESUMEN}

Las ciencias de comunicación pueden ayudar a mostrar a las Iglesias como llegar al hombre. El contexto social y la situación de vida de cualquier persona, condiciona y influencia la comunicación de la Iglesia con el pueblo. Para que el mensaje cristiano tenga acceso y puede ser entregado al pueblo, las Iglesias deben reconocer las necesidades y deseos especîficos de este pueblo. Bajo este aspecto se puede desarrollar y definir una tipologîa del público. Finalmente, las Iglesias deberîan no solamente tratar de ser comprensibles en sus mensajes, sino que además deberîan estar abiertas en tanto que emisor a los deseos del receptor y estar concientes del interés de sus mensajes.

Estas medidas determinan en gran parte el impacto del mensaje cristiano. 